\title{
Universidad Nacional de Rosario: Análisis de la experiencia del acompañamiento pedagógico virtual durante el 2020, en el marco de la pandemia Covid19
}

A más de un año del inicio de la declaración del aislamiento social obligatorio, en el primer período, y del distanciamiento social obligatorio vigente en la actualidad, producto de la pandemia Covid19, nos proponemos en este trabajo presentar las principales acciones llevadas adelante por nuestra Universidad para afrontar el proceso de enseñanza-aprendizaje en tan difícil contexto.

La suspensión del dictado de clases definido por resolución del Ministerio de Educación y la declaración del aislamiento social obligatorio a partir del 20 de marzo de 2020, decretado por el gobierno nacional, nos encontró, como Universidad, en un proceso de implementación de políticas promovidas con fuerte decisión a partir de la asunción de la actual gestión de gobierno, ocurrida en agosto de 2019.

En este sentido, ordenaremos esta presentación en base a dos dimensiones centrales: a) lineamientos generales de funcionamiento que permitieron establecer criterios y procedimientos comunes al conjunto de las distintas unidades académicas; b) recursos educativos y tecnológicos implementados en la modalidad virtual y políticas de apoyo a docentes y estudiantes.

\section{A. Lineamientos generales de funcionamiento}

La Universidad Nacional de Rosario (UNR) conformó su Comité de Contingencia, cuyo primer documento, con fecha domingo 15 de marzo de 2020, fue el resultado del consenso alcanzado entre los integrantes del equipo de gestión, decanos y decanas de las 13 unidades académicas, directoras y director de las tres escuelas medias dependientes de la UNR y representantes gremiales de docentes, nodocentes y estudiantes. Allí se definió posponer el inicio de las actividades académicas hasta el $1^{\circ}$ de abril y se delegó en el área Académica y de Aprendizaje la elaboración de lineamientos comunes para el funcionamiento de las unidades académicas.

Las actividades a implementar se definieron como "acompañamiento pedagógico virtual", entendiendo como prioritario sostener el vínculo, en tiempos de aislamiento preventivo, social y obligatorio que demandaba políticas concretas de acompañamiento y cuidado hacia nuestras y nuestros estudiantes. El término "acompañamiento pedagógico virtual" buscó diferenciar las acciones encaradas de lo que se define como educación a distancia, en términos de modelo pedagógico. Siempre entendimos que tanto para docentes como para estudiantes el desafío solo era posible concretarlo si partíamos de reconocer que el esfuerzo no implicaba

\footnotetext{
* Coordinadora del Área Académica y de Aprendizaje, Universidad Nacional de Rosario (UNR, Argentina). Correo electrónico: cevoras@hotmail.com
} 
convertir carreras presenciales en carreras a distancia. El desafío era contener a las y los estudiantes en espacios formativos adaptados a una realidad distinta, cuyo impacto trasciende ampliamente el contexto educativo.

El resultado de estas tareas fueron resoluciones decanales, resoluciones de consejos directivos, según el caso, que delinearon con la especificidad propia de cada realidad, las modalidades de funcionamiento tanto para la conformación de las aulas como para la eventual toma de exámenes, según lo dispusiera cada Facultad y, en su interior, cada cátedra.

La UNR cuenta con 12 Facultades y un Centro de Estudios Interdisciplinarios, dedicado exclusivamente a carreras de posgrado, que comprenden 350 carreras, en los distintos niveles. Esta realidad requirió contemplar la pluralidad de situaciones desde el punto de vista de las propuestas formativas, las actividades prácticas, las localizaciones territoriales, el plantel docente, el acceso a la información, entre otras cuestiones, para la toma de decisiones. Por ello descentralizar hacia las unidades académicas las lógicas de construcción de consensos y las lógicas resolutivas fue un posicionamiento intencional, cuyo principal propósito fue respetar la diversidad, las necesidades, los límites y las oportunidades específicos y propios de cada realidad disciplinar, organizacional e institucional.

\section{B. Recursos educativos y tecnológicos implementados en la modalidad virtual y políticas de apoyo a docentes y estudiantes}

De los recursos educativos. El desarrollo de las actividades de acompañamiento pedagógico virtual implementadas respondió a las características que nuestra Universidad posee en relación con la existencia de distintas plataformas virtuales que anteceden a este contexto mundial de pandemia e, incluso, a la conformación en 2018 del Sistema Institucional de Educación a Distancias (SIED-UNR). Por ello la información y el análisis que a continuación brindaremos refiere específicamente al Campus Virtual UNR, en el cual se inscriben ocho unidades académicas, un conjunto de aulas de otras dos Facultades y las escuelas medias.

El Campus Virtual UNR utiliza Moodle, una plataforma de aprendizaje de distribución libre, de código abierto. El espacio de Comunidades, dentro del Campus Virtual, se conformó hace varios años atrás, para brindar un acompañamiento físico-virtual a docentes, investigadores y estudiantes de la UNR, destinado a complementar las actividades presenciales y así ha sido utilizado durante años anteriores con esta finalidad. Para tener una dimensión de su evolución, a inicios de 2008 Comunidades tenía 20 espacios virtuales; en marzo de 2016 tenía 665 espacios activos. A la fecha Comunidades cuenta con más de 5.200 espacios activos, de los cuales 2.900 aproximadamente fueron creados desde el 15 de marzo de 2020 en adelante. Por lo tanto, Comunidades se constituyó en el espacio virtual en el que se insertaron las actividades académicas de pregrado, grado y posgrado desarrolladas durante este período de pandemia.

Asimismo, se potenciaron y ampliaron los tutoriales para docentes y estudiantes disponibles en la Plataforma. También se diseñó y puso en funcionamiento un curso on line, continuo, de capacitación para docentes, el que contó con la participación de más de 950 docentes. Además de los módulos que se implementaron a distancia, también se ofrecieron encuentros diarios sincrónicos (mañana, tarde y noche) coordinados por personal del Campus a los fines de abordar temáticas específicas, de carácter individual o a pedido de las unidades académicas.

Por otra parte, se generaron cuentas de Meet educativo, bajo el dominio uvr (Universidad Virtual Rosario), para todas las unidades académicas y escuelas medias a los fines de facilitar el desarrollo de actividades sincrónicas por fuera de la plataforma. Este recurso fue muy bien recibido por los equipos de gestión y las y los docentes y, en algunas Facultades, fue el más utilizado para el dictado de clases.

La posibilidad de sostenimiento de todas estas actividades, en un contexto vertiginoso, complejo, incierto y angustiante, fue posible por el alto compromiso de todo el equipo permanente de trabajo del Campus Virtual y el equipo de Tecnologías, y de las y los representantes de las 
unidades académicas que integran el Sistema Institucional de Educación a Distancia (SIEDUNR).

En términos del volumen de concurrencia a aulas virtuales, considerando las que están registradas en el Campus Virtual y las que se desarrollan en otras plataformas, el promedio de ingreso diario es desde abril de 2020 de 40.000 usuarios, comprendiendo allí a estudiantes y docentes.

De los recursos tecnológicos. La decisión de continuar con el calendario académico y emplear el Campus como recurso principal implicó asumir el desafío de adecuar en pocos días la plataforma, para que soportara la masividad de alumnos y el volumen de contenidos. Esto significó un cambio estructural muy importante ya que pasamos de un sistema monolítico en el cual en un servidor residía toda la plataforma a un sistema distribuido que debía soportar una alta concurrencia. El cambio consistió en separar en granjas de servidores a dos de las unidades académicas más grandes, Ciencias Médicas y Ciencias Económicas; a posteriori conformamos granjas con Psicología y Humanidades y Artes; el resto de las unidades académicas permanecieron en una misma plataforma. Para lograr sostener estos cambios de gran envergadura se procedió a la adquisición de hardware que pudiera soportar la demanda. Por lo tanto, hoy la UNR cuenta con 5 plataformas para el desarrollo de las actividades académicas.

De las políticas de apoyo a docentes y estudiantes. La Universidad trabajó para un abordaje integral del proceso de acompañamiento pedagógico virtual, reconociendo que las desigualdades en la distribución de los recursos están siempre presentes en la estructura social y, por lo mismo, en el sistema educativo. Por ello, parte de esas desigualdades, sobre todo las vinculadas con comedores universitarios, acceso a material de estudio y el acceso a recursos tecnológicos, cobraron mayor visibilidad durante esta experiencia inédita para nuestra sociedad. El área de Bienestar Universitario promovió políticas focalizadas para atender estas necesidades. Desarrolló una línea de becas Conectar que significó una fuerte inversión de recursos económicos. Ello permitió otorgar más de 800 módems a estudiantes y 120 a docentes que carecían de conectividad, y tienen asegurado ese servicio por un año con la cantidad de datos suficiente para el desarrollo de actividades académicas virtuales como así también acciones administrativas de modo remoto.

Otro recurso que se desarrolló para garantizar el acceso a recursos audiovisuales fue la plataforma de producciones audiovisuales de apoyo educativo (https://educom.unr.edu.ar/). Es un portal de materiales producidos por docentes e investigadores de la UNR, puestos a disposición de la comunidad universitaria, tanto de nuestra propia institución como de cualquier otra que quiera utilizarlos con fines educativos.

Otro aspecto a considerar, de manera especial, fue el seguimiento de los ingresantes 2020. A tal fin la UNR creó la Plataforma Ingresantes 2020. En este portal los ingresantes contaron con conversatorios, objetos digitales educativos acerca de la vida universitaria, un espacio virtual de orientación pedagógica, tutorías de pares virtuales, consultas por whatsapp, entre otros recursos. El objetivo fue trabajar a través de encuentros individuales y colectivos con los ingresantes 2020 y todos aquellos estudiantes que necesitaran apoyo para afrontar los requerimientos de la vida académica universitaria.

En esta misma línea, comenzó a planificarse el ingreso 2021, considerando que sería bajo modalidad virtual, y destinado a estudiantes que a lo largo de 2020 no habían asistido a los establecimientos educativos de enseñanza media. Se avanzó en promover una modalidad de ingreso que contemplara el desarrollo de una prueba piloto de un curso pre-universitario que permitiera establecer un vínculo con las personas pre-inscriptas a las carreras a través de 3 módulos desarrollados con equipos docentes de la Universidad y el equipo de Campus Virtual: a. Módulo de Vida Universitaria; b. Módulo de Lectura y Escritura Académica en la Universidad; y c. Módulo de Lógica para Pensar. Estos espacios formativos se complementaron con otras dos acciones relevantes: 1. El Programa Codo a Codo, en el que se incluyeron 330 tutores y tutoras pares a través de una modalidad de beca por concurso, esto es estudiantes avanzados de las 
carreras de la Universidad, para que contribuyeran a facilitar la transición de los ingresantes de la escuela media a la Universidad. Esto implicó más que triplicar el número de tutores con que habitualmente trabajaba la Universidad; y 2. La conformación del Patio Virtual, espacio desarrollado en la plataforma Discord (herramienta utilizada para video juegos), para conformar un ámbito de circulación de las y los ingresantes y tutoras y tutores pares que se constituyera en el lugar de encuentro para compartir problemas comunes, inquietudes y canalizar dudas. Por los módulos y el Patio Virtual transitaron más de 9.000 ingresantes a la UNR.

\section{Algunas conclusiones}

Los intensos meses de actividad en el marco de pandemia no pueden ser analizados sin tener en consideración la afectación de las subjetividades que atravesamos y seguimos atravesando integrantes de equipos de gestión, docentes y estudiantes. Esa realidad quedó plasmada en los resultados de la encuesta realizada en marzo 2021 cuyo análisis no es posible incluir en este trabajo. Al respecto sólo nos permitiremos mencionar que incertidumbre, angustia, estrés, malestar son términos presentes en las respuestas de docentes y estudiantes cuando refirieron a los problemas socio-afectivos que debieron enfrentar en 2020. También hay una fuerte relación con los problemas de conectividad y de acceso a dispositivos. En ese marco, asimismo las expectativas a futuro dan cuenta de que esta experiencia debe ser un paso hacia la inclusión de nuevas modalidades educativas, basadas en el uso de tecnologías y de recursos de la educación a distancia, en el nivel universitario.

Por ello creemos que nuestro balance institucional debe recoger aprendizajes y desafíos:

a. Fortalecimiento de la tarea en equipo: logramos una articulación de políticas y de acciones que se plasmaron en decisiones que involucraron a diversos actores y áreas de la Universidad.

b. Doble desafío a futuro: avanzar en el tránsito del acompañamiento pedagógico virtual a la educación a distancia, y en el tránsito a nuevas formas de la educación presencial: si bien nuestras carreras de grado son 100\% presenciales, esta experiencia abrió el reconocimiento a la "devaluada" educación a distancia. Lo cierto es que ya la educación a distancia no será la misma y tampoco la educación presencial podrá ignorar nuevas formas de vinculación, mediadas por tecnologías.

c. Reconocer las limitaciones del orden socio-económico que no puede resolver la Universidad pero que deben ser objeto de políticas públicas nacionales y por las que la universidad pública debe bregar en los distintos ámbitos de decisión: acceso a la conectividad y a dispositivos que garanticen la tarea de enseñar y aprender.

d. Toda esta enorme tarea no podría haberse logrado sin la decisión política de garantizar que la Universidad cumpliera con su función social y educativa, mucho más aún en un momento de la historia de la humanidad tan peculiar, tan incierto, tan desafiante. Aquí va el reconocimiento a todos y cada uno de los integrantes de la comunidad de la UNR que cotidianamente asumieron este compromiso con dificultades, miedos, en condiciones familiares adversas, pero con una fuerte convicción de que la Universidad no cerrara sus puertas, aunque las físicas no estuvieran habilitadas.

TRABAJO RECIBIDO: O1/06/2021 